\title{
Cuerpos extraños radiopacos con doble contorno en la vía digestiva: ison siempre pilas de botón?
}

\section{Are strange radiopaque bodies with double contours in the digestive tract always button cell batteries?}

Alexis José Ormeño Julca, MD, ${ }^{* *}$ Nora Flor Echevarría López, MD. ${ }^{2}$

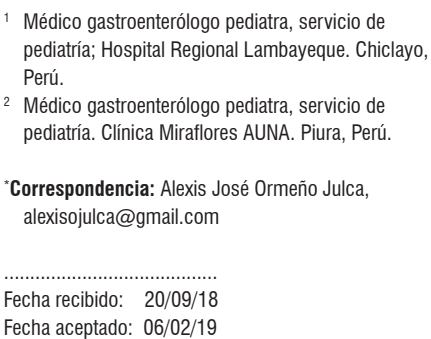

\begin{abstract}
Resumen
La ingesta accidental de cuerpos extraños constituye una emergencia en los servicios de atención pediátrica y el grupo más frecuentemente afectado se encuentra en los pacientes entre los 6 meses y los 3 años. Asimismo, la ingesta de pilas de botón es un factor de riesgo importante para el desarrollo de complicaciones tempranas severas como la perforación esofágica y mediastinitis, y a largo plazo, la aparición de estenosis.

Por este motivo, su identificación temprana y la extracción constituyen las estrategias más importantes de manejo. Se ha descrito como característico el hallazgo del doble contorno en la radiografía frontal cérvicotoracoabdominal en estos pacientes. Sin embargo, se ha reportado el mismo signo radiológico en cuerpos extraños diferentes. En el presente artículo se presentan dos casos con estas características.
\end{abstract}

\section{Palabras clave}

Pila, botón, doble contorno, radiografía.

\begin{abstract} characteristics.

Keywords

Battery, button cell, double contour, radiography.
\end{abstract}

Accidental ingestion of foreign bodies constitutes an emergency for pediatric care services especially since the group most frequently affected consists of children between 6 months and 3 years of age. Ingestion of button cell batteries has high risks for severe early complications such as esophageal perforations and mediastinitis, as well as the long term risk of stenosis. Early identification and extraction are the most important management strategies. A double contour on a frontal cervical, thoracic, or abdominal $\mathrm{x}$-ray is characteristic, but this same radiological sign has been reported for other foreign bodies. We present two cases with these

\section{INTRODUCCIÓN}

La ingesta accidental de cuerpos extraños constituye una emergencia en los servicios de gastroenterología, pediatría y cirugía pediátrica. Los problemas asociados con el tracto digestivo suelen ser benignos. Sin embargo, algunos de estos objetos pueden producir lesiones graves en el esófago $(1,2)$. Del total de casos, en la población pediátrica se reporta el 60-80\%, con una incidencia máxima entre los 6 meses y los 3 años (3). Los cuerpos extraños más frecuentemente registrados son monedas (50-70 \% de los casos) (4-6); los objetos afilados como aretes, tachuelas, alfileres, agujas y huesos (33\%), y las baterías de botón (15\%) (4).

Los estudios radiológicos son de utilidad para el diagnóstico y la ubicación de los cuerpos extraños. No obstante, se reporta que solo un $65 \%$ de estos son radiopacos $(7,8)$. En este sentido, cobra importancia realizar la diferenciación entre la ingestión de una moneda y de una pila de botón, 
dadas sus características radiológicas similares y los distintos requerimientos terapéuticos que implican (9).

En la radiografía frontal cérvico-toracoabdominal se ha descrito la presencia del signo de doble contorno como confirmatorio en los casos de pilas de botón impactadas en el esófago (10). Por tanto, se presentan dos casos de pacientes pediátricos que tuvieron esta característica radiológica, pero con la ingesta de cuerpos extraños diferentes.

\section{CASO 1}

Paciente varón de 4 años, natural de Piura, que ingresó por el servicio de emergencia del Hospital Santa Rosa de Piura, con el antecedente de ingesta de un cuerpo extraño, un día antes. A su ingreso, cursó con disfagia y sialorrea. Durante el examen físico, se encontró hemodinámicamente estable. Las pruebas de laboratorio no estuvieron alteradas y en la radiografía de tórax se evidenció un cuerpo extraño radiopaco y redondeado en el esófago superior, con doble contorno (Figura 1A). El paciente fue evaluado por el servicio de cirugía y se decidió su programación para la extracción con una sonda de Foley. Al terminar el procedimiento, se obtuvo un botón metálico de pantalón (Figura 1B). No se presentaron complicaciones después de la intervención y el paciente fue dado de alta con buena tolerancia oral.

\section{CASO 2}

Paciente varón de 1 año y 10 meses, natural de Chiclayo, que ingresó por el servicio de emergencia del Hospital Regional
Lambayeque de Chiclayo, con el antecedente de ingesta de un cuerpo extraño desconocido, $13 \mathrm{~h}$ antes. En principio, el paciente presentó una dificultad respiratoria que se resolvió espontáneamente. Luego de ello, se observaron vómitos e hiporexia. Asimismo, en el momento de ingreso se evidenció la existencia de sialorrea y disfagia. Durante el examen físico se halló hemodinámicamente estable y no se presentaron alteraciones en el hemograma ni en la bioquímica.

En la radiografía de tórax se observó un cuerpo extraño redondeado y radiopaco en el esófago superior, con doble contorno (Figura 2A). Ante la incertidumbre sobre el tipo de cuerpo extraño ingerido, y dada la sospecha de una pila de botón, se procedió a su inmediata programación para endoscopía bajo anestesia general. Finalmente, se extrajo una moneda (Figura 2B). El paciente toleró el procedimiento en forma adecuada y, luego de probarse vía oral, fue dado de alta.

\section{DISCUSIÓN}

En los pacientes de pediatría con cuerpos extraños ingeridos es indispensable el diagnóstico precoz y la extracción inmediata (idealmente dentro de las primeras $2 \mathrm{~h}$ ) de las pilas de botón, ya que están relacionadas con complicaciones tempranas y usualmente severas (perforación esofágica, mediastinitis y fistula traqueoesofágica) y con complicaciones tardías, como en casos de desarrollo de estenosis esofágica $(11,12)$.

Si bien el signo del doble contorno en la radiografía frontal es característico de las pilas de botón, no es exclusivo de estos cuerpos extraños. Así lo reportan Gan y colaboradores (13), quienes documentaron el caso de una paciente

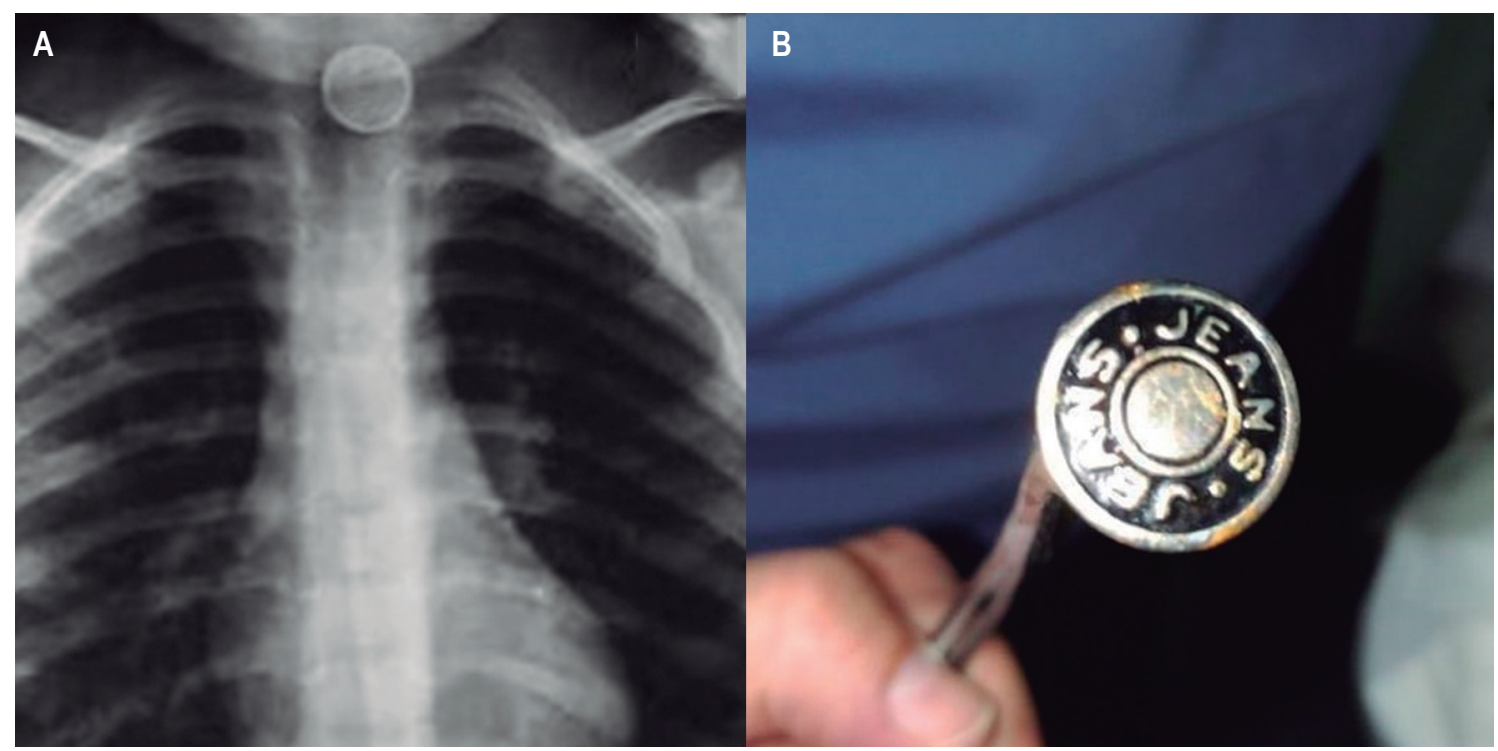

Figura 1. A. Radiografía posteroanterior de tórax que muestra un cuerpo extraño radiopaco y redondeado en el tercio superior del esófago, con un doble contorno. B. Botón metálico de pantalón extraído con una sonda de Foley. 


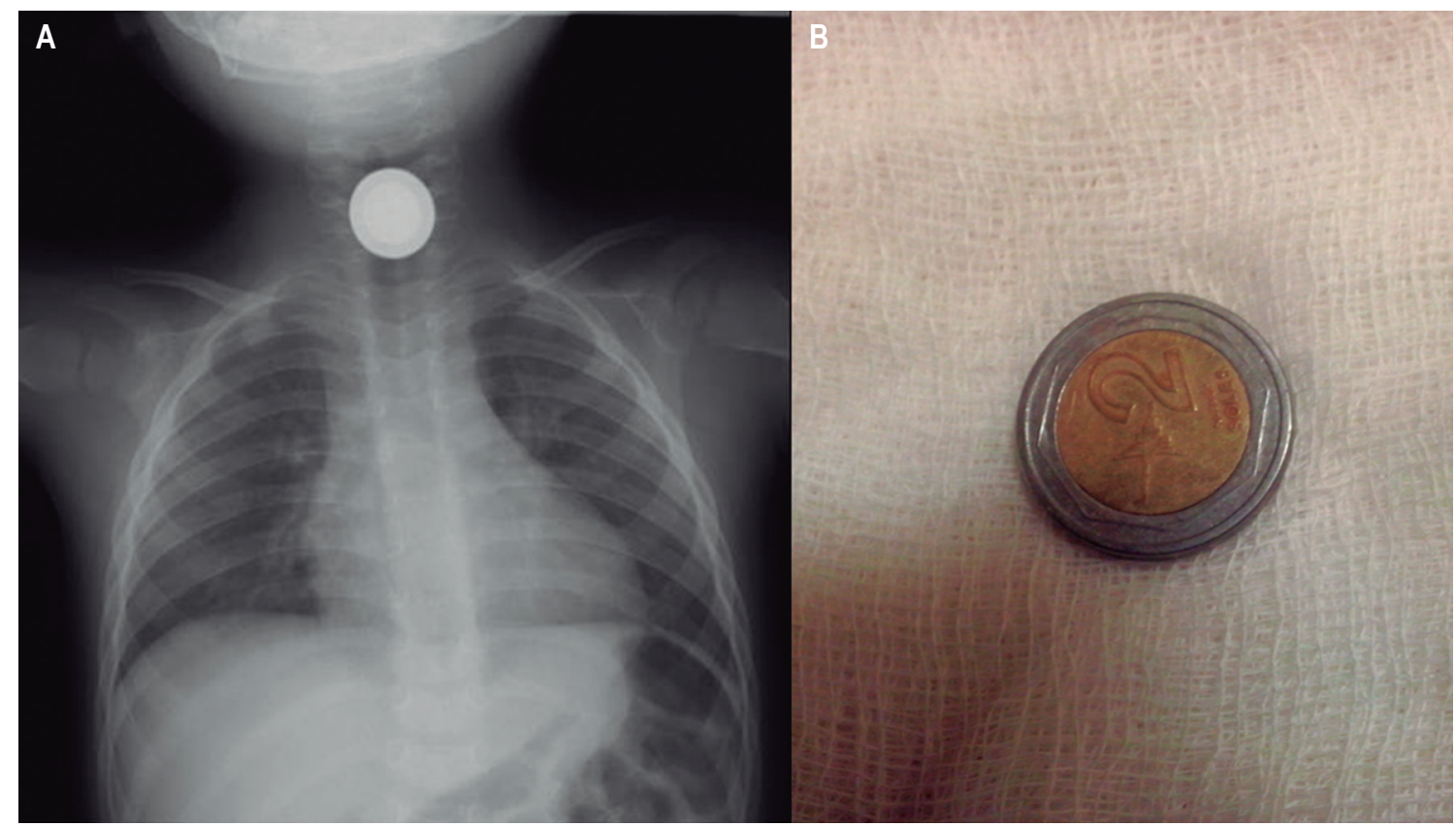

Figura 2. A. Radiografía anteroposterior de tórax que muestra un cuerpo extraño radiopaco y redondeado en el tercio superior del esófago, con doble contorno. B. Moneda extraída que corresponde a dos nuevos soles peruanos, los cuales contienen doble aleación y provocan una doble densidad en la radiografía frontal.

con ingesta de dos monedas juntas, impactadas en el esófago. Otro ejemplo de ello es observable en nuestros dos casos ya descritos.

Por tal motivo, se sugiere conocer las medidas de los diámetros de las diferentes monedas y de las baterías de botón que se usan en el medio local. Asimismo, se debe considerar que, en las radiografías, las monedas muestran un borde externo ancho, por lo que al tener una o dos aleaciones se presentan con una densidad uniforme o dos cambios de densidad. Esto las diferencia de las pilas de botón, que muestran un borde delgado y suelen presentar de 3 a 4 cambios de densidad (14).

En consecuencia, se recomienda solicitar siempre una radiografía lateral, en la cual sea posible visualizar la diferencia de diámetro entre el cátodo y el ánodo en el caso de la pila de botón, y a diferencia de la moneda, que muestra un borde uniforme (Figuras 3A y 3B) $(4,15)$.

\section{Conflicto de intereses}

Los autores declaran no tener ningún conflicto de interés.

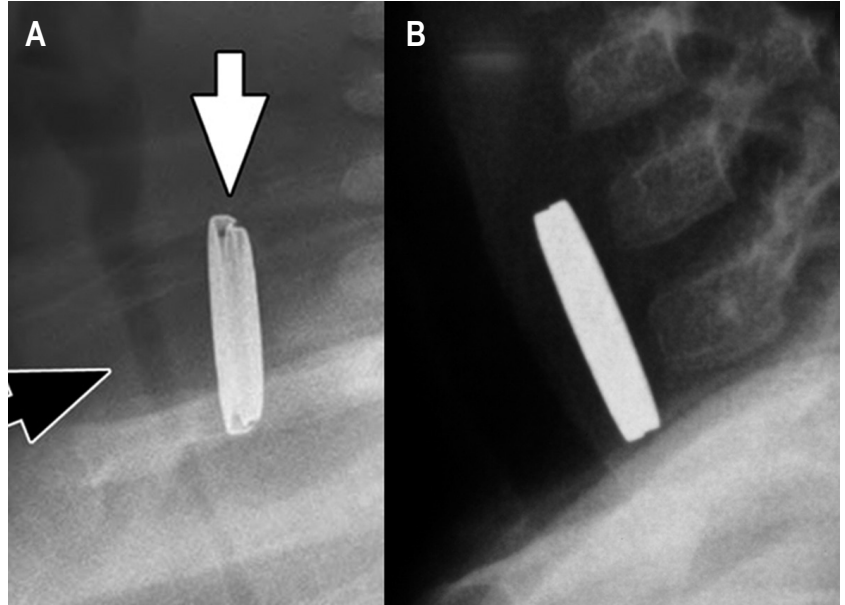

Figura 3. A. Radiografía lateral que muestra claramente la diferencia de diámetro entre el cátodo y el ánodo (flecha blanca), así como las diferentes densidades correspondientes a las distintas aleaciones que se encuentran en las pilas de botón (flecha negra). B. Radiografía lateral de un paciente con ingesta de doble moneda, las cuales se hallan adosadas entre sí, por lo que muestran un borde lateral uniforme y una sola densidad radiológica. 


\section{REFERENCIAS}

1. Guzmán F, Morales JA, Chacín Z. Evaluación, diagnóstico y manejo de los cuerpos extraños en esófago en pacientes pediátricos: experiencia en el Hospital Universitario de Maracaibo, marzo 1996-marzo 2000. Arch Venez Pueri Pediatr. 2001;64(3):148-152.

2. Barber GB, Peppercorn MA, Ehrlich C, Thurer R. Esophageal foreign body perforation: report of an unusual case and review of the literature. Am J Gastroenterol. 1984;79(7):509-11.

3. Kay M, Wyllie R. Pediatric foreign bodies and their management. Curr Gastroenterol Rep. 2005;7(3):212-8. https:// doi.org/10.1007/s11894-005-0037-6

4. Ormeño A, Florián A. Características clínicas y epidemiológicas de los pacientes atendidos con cuerpos extraños en vía digestiva en el Instituto Nacional de Salud del Niño desde Enero del 2009 hasta Diciembre del 2011. Rev Peru Pediatr. 2013;66(2):51-61.

5. Arana A, Hauser B, Hachimi-Idrissi S, Vandenplas Y. Management of ingested foreign bodies in childhood and review of the literature. Eur J Pediatr. 2001;160(8):468-72. https://doi.org/10.1007/s004310100788

6. ChengW, Tam PK. Foreign-bodyingestion in children: experience with 1,265 cases. J Pediatr Surg. 1999;34(10):14726. https://doi.org/10.1016/S0022-3468(99)90106-9

7. Brady PG, Johnson WF. Removal of foreign bodies: the flexible fiberoptic endoscope. South Med J. 1977;70(6):702-4. https://doi.org/10.1097/00007611-197706000-00019

8. Daza W, Sánchez E, Urueña M. Urgencias pediátricas: cuerpos extraños en el tracto digestivo alto. Temas pediátricos 2008;25(1):52-56.
9. Jatana KR, Litovitz T, Reilly JS, Koltai PJ, Rider G, Jacobs IN. Pediatric button battery injuries: 2013 task force update. Int J Pediatr Otorhinolaryngol. 2013;77(9):1392-9. https:// doi.org/10.1016/j.ijporl.2013.06.006

10. Bakshi SS. Button Battery Ingestion. Balkan Med J. 2018;35(2):212-213. https://doi.org/10.4274/balkanmedj.2017.0523

11. Ettyreddy AR, Georg MW, Chi DH, Gaines BA, Simons JP. Button battery injuries in the pediatric aerodigestive tract. Ear Nose Throat J. 2015;94(12):486-93. https://doi. org/10.1177/014556131509401207

12. Fuentes S, Cano I, Benavent MI, Gómez A. Severe esophageal injuries caused by accidental button battery ingestion in children. J Emerg Trauma Shock. 2014;7(4):316-321. https://doi.org/10.4103/0974-2700.142773

13. Gan RW, Nasher O, Jackson PB, Singh SJ. Diagnosis of button battery ingestion by 'halo' radiographic sign: an exception to the rule. BMJ Case Rep. 2015;2015:bcr2015209908. https://doi.org/10.1136/bcr-2015-209908

14. Blanco-Rodríguez G, Penchyna-Grub J, Ochoa-Guajardo PL, Álvarez-Neri H, Porras-Hernández JD, Teyssier-Morales G. ¿Qué tan urgente es extraer una pila de disco alojada en el esófago? Bol Med Hosp Infant Mex. 2008;65(4):282-289.

15. Pugmire BS, Lin TK, Pentiuk S, de Alarcon A, Hart CK, Trout AT. Imaging button battery ingestions and insertions in children: a 15-year single-center review. Pediatr Radiol. 2017;47(2):178-185. https://doi.org/10.1007/s00247016-3751-3 